\title{
A Composite Weight Based Access Network Selection Algorithm in Marine Internet
}

\author{
Liang Zhou $^{1,2}$, Shengming Jiang ${ }^{1}$ \\ 1 College of Information Engineering, Shanghai Maritime University, \\ Shanghai, China 201306 \\ ${ }_{2}$ Network \& Information Center, Shanghai Municipal Educational Examinations Authority, \\ Shanghai, China 200433 \\ lzhoulshmeea.edu.cn
}

\begin{abstract}
The $21^{\text {st }}$ century is the ocean century, and ocean informatization is one of the key requirements. Marine Internet (MI) is the basic infrastructure of ocean informatization, so developing the MI technology is of great significance. There are several access networks maybe available in a MI system, and a network user has to choose the best one to support the application. The selection scheme needs to consider may factors in order to make a smart decision, which is a multi-index decision problem. These factors include user preferences, business types, and the performance as well as cost of alternative access networks. Combining with the Analytic Hierarchy Process (AHP) algorithm and entropy theory, we develop a method for the user to select access networks in a smart way. It adopts subjective and objective weights and a utility function to select the target access network. The simulation results carried out on the Exata platform show that the proposed scheme can improve the network performance with reduced cost.
\end{abstract}

Keywords: Marine Internet; Access method; Analytic Hierarchy Process(AHP); Entropy; Exata simulation

\section{Introduction}

The $21^{\text {st }}$ century is the ocean century, and ocean informatization is one of the key requirements. Marine Internet (MI) is the basic infrastructure of ocean informatization, so developing the MI technology is of great significance In the marine Internet, there are multiple access networks maybe available for selection, which typically include shore-based networks, self-organizing ship networks, high-altitude communication platforms, satellite networks and so on[1]. Network users often need to choose one from a number of access networks to support the current application, considering the user preferences, business type and the performance as well as cost of alternative access networks and other factors.

When multiple access networks are available, a network selection is usually based on a mathematical algorithm that gives a weight to each metric. With the utility function, the comprehensive utility value of each index corresponding to each alternative network is calculated. Finally, the access network with the largest utility value is selected as the best solution. The problem with such kind of algorithm is that the 
assignment of weights is dependent on the subjective experience of the operator as mentioned in [2], which then proposed an algorithm based on the combination of subjective and objective weight to determine the weight of the network attributes, taking into account the user preferences and different business types and Quality of Service(QoS). The algorithm works well in a dense user scenario. However, the marine Internet is characterized by sparse distribution of users, which mainly consist of ships. So far, such kind of scenario has not been investigated in the literature. Therefore, this paper combines the Analytic Hierarchy Process (AHP) [3] and the entropy theory to set the subjective and objective weights respectively, and then selects the target network through the utility function.

\section{Access Network Selection Algorithm Based on Compound Weights}

\subsection{Framework of the proposed algorithm}

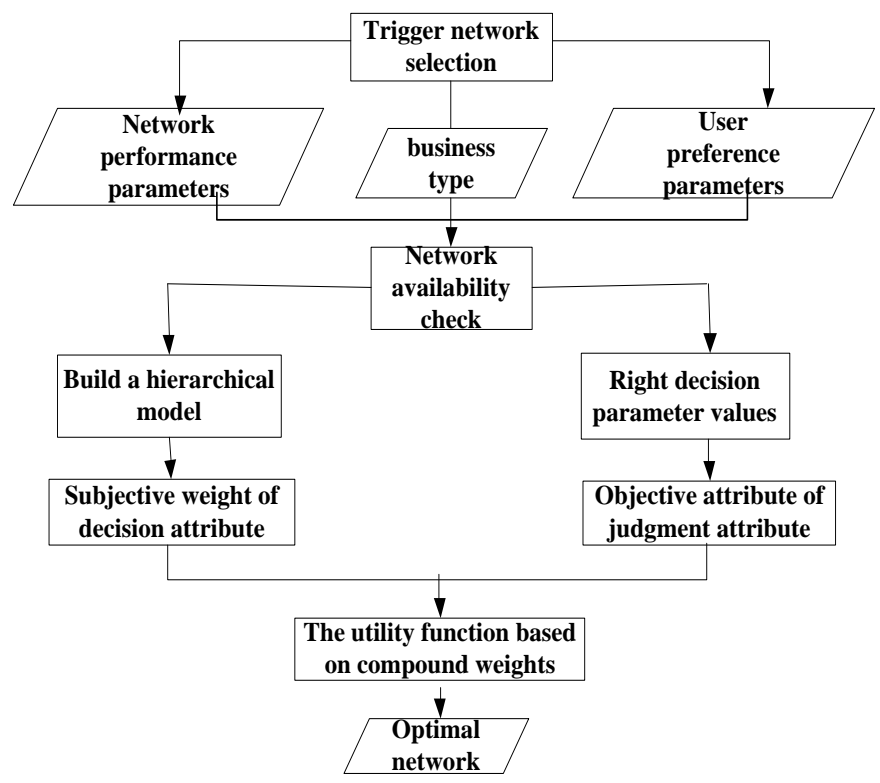

Fig. 1. Framework of the proposed network selection algorithm. It shows the framework of the algorithm. The Analytic Hierarchy Process (AHP) is an effective method to solve the complex multi-index decision problem, and the entropy weight method has objective fairness and can judge the change of the judgment index objectively[4]. Therefore, this paper combines these two algorithms to deal with the access network selection in the marine Internet. The algorithm considers the network performance requirements and user preferences, makes the algorithm have a good applicability. 


\subsection{Network Selection Model Based on Analytic Hierarchy Process}

Many factors affect the heterogeneous network selection, and it is unrealistic to choose an optimal network without considering them comprehensively. We can focus on several common, important factors as an indicator. The users of the same business costs have different tendencies, and some hope to have high-quality services, and some with a pursuit of economic benefits. Furthermore, different types of applications have different basic requirements of network performance. For example, for marine emergency search and rescue applications, the network needs to ensure the stability of communication and timeliness. Based on the above considerations, the AHP algorithm is used to establish the hierarchical model. With consideration of the user preference in the criterion layer, the sub-criterion layer considers the application requirements of the network type.

\section{1) Hierarchical Model of Network Selection Based on User Preference}

As shown in Fig. 2, first, select the QoS level $(\alpha)$, the network available load ( $\beta$ ) and the price charge $(\gamma)$ as the network performance indicators, and then combine them with the user's network performance bias, QoS priority mode and price priority mode.

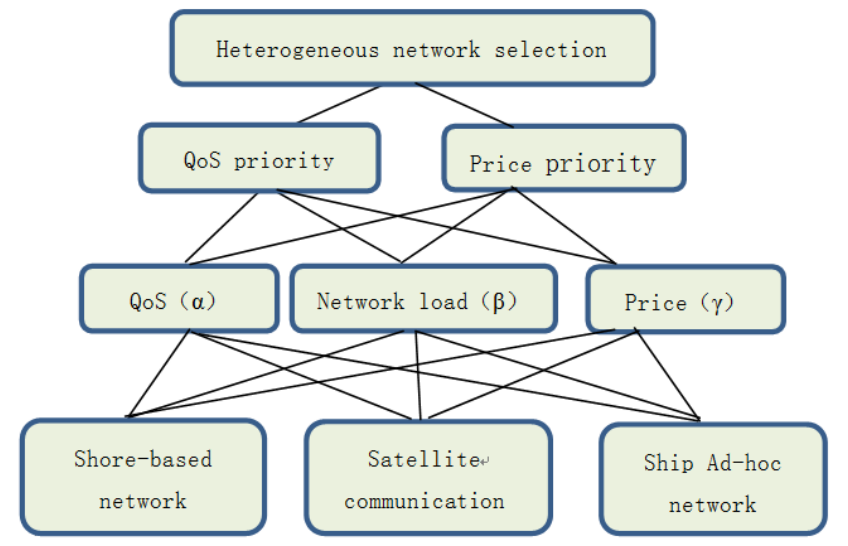

Fig. 2. The hierarchical model based on the user's preferred access network. Users in the QoS priority mode in the access network selection give priority to application requirements with an inclination to the performance of the access network an acceptable price. Under this strategy, the priority of the three network attribute indicators is as follows: QoS level $>$ network load $>$ price. Users in the price priority mode is more concerned about the cost of the access network, while the sensitivity of the network performance is low. Then the three network attribute indicators priority is price $>$ QoS level $>$ network load.

We first establish the two models of the decision matrix, set the two indicators of the importance of each in Table 1, where the values depend on the experience of the decision maker, and can be appropriately adjusted to suit the specific needs of the algorithm.

Table 1. Setting for different strategies under the decision matrix 
(a)QoS priority

\begin{tabular}{l|clc}
\hline QoS priority & QoS level $(\alpha)$ & Load $(\beta)$ & Price $(\gamma)$ \\
\hline QoS level $(\alpha)$ & 1 & 2 & 4 \\
Load $(\beta)$ & $1 / 2$ & 1 & 2 \\
Price $(\gamma)$ & $1 / 4$ & $1 / 2$ & 1 \\
\hline
\end{tabular}

(b)Price priority

\begin{tabular}{l|cll}
\hline Price priority & QoS level $(\alpha)$ & Load $(\beta)$ & Price $(\gamma)$ \\
\hline QoS level $(\alpha)$ & 1 & 2 & $1 / 2$ \\
Load $(\beta)$ & $1 / 2$ & 1 & $1 / 4$ \\
Price $(\gamma)$ & 2 & 4 & 1 \\
\hline
\end{tabular}

The judgment matrix is sorted and the consistency is verified. The maximum Eigen values of the two matrices are calculated as 3 , and the calculated values are all zero. Note that the settings of the matrix conform to the consistency requirements. Then, the weights of the indexes in the two modes are obtained and normalized, and the calculated values are all four decimal places.

$$
\left\{\begin{array}{c}
\mathrm{W}_{\mathrm{QoS}}=\left\{\omega_{\alpha}, \omega_{\beta}, \omega_{\gamma}\right\}=\{0.5714,0.2857,0.1429\} \\
\mathrm{W}_{\text {Price }}=\left\{\omega_{\alpha}, \omega_{\beta}, \omega_{\gamma}\right\}=\{0.2857,0.1429,0.5714\}
\end{array}\right.
$$

2) QoS hierarchical model based on service type

There are many types of network applications with different network service quality requirements. For example, at present, 3GPP is divided into four categories according to the different QoS features of network application: session class, streaming class, interactive class and background class service [7].

Different services corresponding to the network performance indicators have different requirements. Therefore, we use QoS $(\alpha)$ in Figure 3 as the target layer of the criterion layer to establish the QoS hierarchical model based on the service type. The quasi-side layer is divided into session class (T1), streaming media class (T2), interactive class (T3), and background class(T4). The subordinate criterion layer includes four parameters: delay $\left(\alpha_{1}\right)$, jitter $\left(\alpha_{2}\right)$, packet loss rate $\left(\alpha_{3}\right)$ and rate $\left(\alpha_{4}\right)$. Take Session class (T1) as an example, the network performance indicators between the decision matrixes are set as shown in Table 2.

Table 2.Judgment matrices based on various business types

Session class - T1

\begin{tabular}{l|llll}
\hline & $\alpha 1$ & $\alpha 2$ & $\alpha 3$ & $\alpha 4$ \\
\hline $\operatorname{Delay}(\alpha 1)$ & 1 & 1 & 3 & 7
\end{tabular}




\begin{tabular}{l|llll}
\hline Jitter $(\alpha 2)$ & 1 & 1 & 3 & 7 \\
Packet loss & $1 / 3$ & $1 / 3$ & 1 & 5 \\
rate $(\alpha 3)$ & $1 / 7$ & $1 / 7$ & $1 / 5$ & 1 \\
TxRate $(\alpha 4)$ & & & \\
\hline
\end{tabular}

According to the AHP algorithm, the maximum Eigen values of the decision matrix corresponding to the four business types are calculated, which are 4.0735, $3.8480,4$ and 4 , respectively. The four decision matrices pass the consistency test; then calculate the QoS sub-parameters based on different types of business weights as listed in Table 3.

$$
\mathrm{W}_{\mathrm{Q}_{\mathrm{O}} \_}=\left\{\omega_{\mathrm{Q} \_\alpha 1}, \omega_{\mathrm{Q} \_\alpha 2}, \omega_{\mathrm{Q} \_\alpha 3}, \omega_{\mathrm{Q}_{\_} \alpha 4}\right\} \quad \mathrm{i} \in\{\mathrm{T} 1, \mathrm{~T} 2, \mathrm{~T} 3, \mathrm{~T} 4\}
$$

Among them, the corresponding weight of the parameters are shown in Table 3.

Table 3. The QoS $(\alpha)$ weights of different service types

\begin{tabular}{l|llll}
\hline Type of service & $\alpha_{1}$ & $\alpha_{2}$ & \multicolumn{1}{c}{$\alpha_{3}$} & $\alpha_{4}$ \\
\hline (T1) & 0.3966 & 0.3966 & 0.16 & 0.0468 \\
(T2) & 0.0615 & 0.2273 & 0.2583 & 0.4529 \\
(T3) & 0.4091 & 0.0455 & 0.4091 & 0.1364 \\
(T4) & 0.0601 & 0.0601 & 0.2647 & 0.6150 \\
\hline
\end{tabular}

3) Ranking the total order and calculating the subjective weight

An overall multi-level hierarchical model based on the network selection problem is drawn in Fig.3, consisting of two modes of QoS priority and price priority target layer in the model, which represent different user preferences. QoS, available network load and price factors are the center of the rule layer, the weights corresponding to the target layer are calculated with Formula 1.In addition, the sub-rule layer under QoS criterion includes delay, jitter, packet loss rate, traffic rate and other factors, also correspond to different business types with different weights.

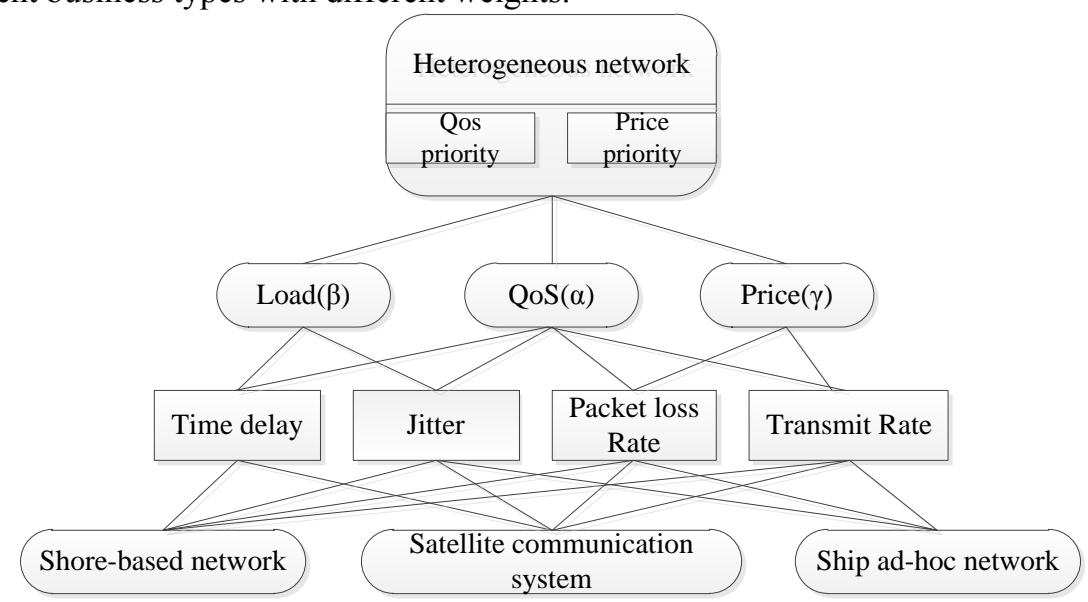

Fig.3. Network selection hierarchy model. The total rank weights are calculated by single rank weight at each level. Formulas 1 and 2 are used to calculate the total order of each index under 
different user preference model, different business types, namely for the time delay $\left(\alpha_{1}\right)$, jitter $\left(\alpha_{2}\right)$, the rate of packet loss $\left(\alpha_{3}\right)$,Rate $\left(\alpha_{4}\right)$,price $(\gamma)$ and subjective weight vector based on different cases: $\mathrm{W}_{1}=\left\{\omega_{\alpha 1}^{\prime}, \omega_{\alpha 2}^{\prime}, \omega_{\alpha 3}^{\prime}, \omega_{\alpha 4}^{\prime}, \omega_{\beta}^{\prime}, \omega_{\gamma}^{\prime}\right\}$

The weight vectors for different scenes are shown in Formulas 3 and 4.

$$
\begin{aligned}
& \text { QoS priority }\left\{\begin{array}{l}
\text { T1: } W_{1}=W_{C 1 \_T 1}=\{0.2266,0.2266,0.0914,0.0267,0.2857,0.1429\} \\
\text { T2: } W_{1}=W_{C 1_{-} T 2}=\{0.0351,0.1299,0.1476,0.2588,0.2857,0.1429\} \\
\text { T3: } W_{1}=W_{C 1 \_T 3}=\{0.2338,0.0260,0.2338,0.0779,0.2857,0.1429\} \\
\text { T4: } W_{1}=W_{C 1 \_T 4}=\{0.0343,0.0343,0.1512,0.3514,0.2857,0.1429\}
\end{array}\right. \\
& \text { Price Priority }\left\{\begin{array}{l}
\text { T1: } W_{1}=W_{C 2_{-} T 1}=\{0.1133,0.1133,0.0457,0.0134,0.1429,0.5714\} \\
\text { T2: } W_{1}=W_{C 2_{-} T 2}=\{0.0176,0.0649,0.0738,0.1294,0.1429,0.5714\} \\
\text { T3: } W_{1}=W_{C 2_{-} T 3}=\{0.1169,0.0130,0.1169,0.0390,0.1429,0.5714\} \\
\text { T4: } W_{1}=W_{C 2_{-} T 4}=\{0.0172,0.0172,0.0756,0.1757,0.1429,0.5714\}
\end{array}\right.
\end{aligned}
$$

After the layered model is built up, should calculate the weight of the single layer firstly, and calculate the total weight of the hierarchy finally. Because the decision matrix is set by the decision-maker itself, it has some limitations.

\subsection{Objective weight based on information entropy}

In order to eliminate the subjective unilateralism of the total weight obtained by AHP, the proposed method uses entropy weight as the objective weight of judgment. Before calculating the entropy weight, the dimensionless decision attributes are introduced, followed by the method of the dimensionless of the decision attributes.

In a decision problem with $m$ alternative network schemes and $n$ network metrics, the given parameter evaluation matrix is:

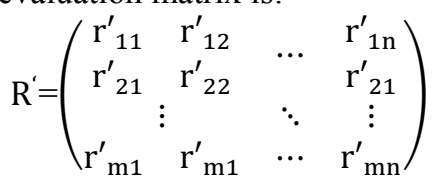

For some network parameter indicators, the smaller the better, such as delay; while for the other, the greater the better, such as speed. In order to describe the changing trend of two kinds of indexes in a unified way, normalization is needed.

The delay $\left(\alpha_{1}\right)$, jitter $\left(\alpha_{2}\right)$ 、 packet loss rate $\left(\alpha_{3}\right)$, rate $\left(\alpha_{4}\right)$, available load $(\beta)$ and network price $(\gamma)$ are chosen as decision criteria in network parameters. Among them, for the delay $\left(\alpha_{1}\right) 、$ jitter $\left(\alpha_{2}\right)$, packet loss rate $\left(\alpha_{3}\right)$ and the network price $(\gamma)$, the smaller the better, and its normalization is: 


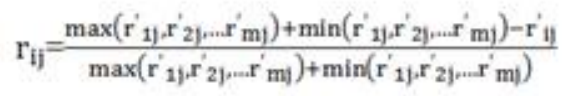

For the rate $\left(\alpha_{4}\right)$ and the available $\operatorname{load}(\beta)$, the bigger the better, and the following formula is used for the normalization processing:

$$
r_{i j}=\frac{r^{\prime}{ }_{i j}}{\max \left(r^{\prime}{ }_{1 j}, r^{\prime}{ }_{2 j}, \ldots r^{\prime}{ }_{m j}\right)+\min \left(r^{\prime}{ }_{1 j}, r^{\prime}{ }_{2 j}, \ldots r^{\prime}{ }_{m j}\right)}
$$

After the parameter is dimensionless, the normalized evaluation matrix $\mathrm{R}$ is obtained:

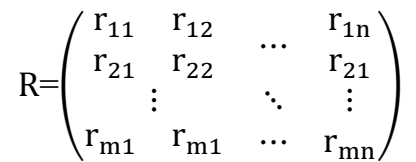

Then calculate the network attributes corresponding to entropy weight, composed of objective weight vector $W_{2}$ as follows:

$$
\mathrm{W}_{2}=\left\{\omega{ }_{\alpha 1}, \omega{ }_{\alpha 2}, \omega{ }_{\alpha 3}, \omega{ }_{\alpha 4}, \omega{ }_{\beta}, \omega{ }_{\gamma}\right\}
$$

\subsection{Utility function}

Selection algorithm is based on a combination of subjective and objective weights from the user perspective and network management. The user preferences and different requirements for network performance by different services are considered firstly. The hierarchical model using the AHP algorithm is built, and the subjective weight of each index is calculated. Then entropy method is used to reflect the dynamic relationship between the various indicators of competition, and reduce the error caused by subjective judgment from the objective standpoint. The selection algorithm is based on the combination of subjective and objective weights, aiming to the process of network selection more reasonably. The algorithm itself is not complicated and is beneficial to generalization. The form of compound weights is as follows:

$$
\begin{aligned}
& \omega_{\mathrm{j}}=\theta \cdot \omega_{\mathrm{j}}^{\prime}+(1-\theta) \cdot \omega_{\mathrm{j}}, \mathrm{j}=\alpha_{1}, \alpha_{2}, \alpha_{3}, \alpha_{4}, \beta, \gamma
\end{aligned}
$$

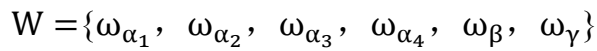

Among them $\theta \in(0,1), \quad \theta=0.5$ in this paper.

For a network with $\mathrm{N}$ candidates, evaluation matrix of the network parameter normalization is $\mathrm{R}=\left(\mathrm{r}_{\mathrm{ij}}\right)_{\mathrm{n} \times 6}$, and we define the utility of each network as follows:

$$
\begin{aligned}
& U=\left[u_{1}, u_{2}, \ldots u_{n}\right]=R \cdot W^{T} \\
& u_{i}=\sum_{j}\left(w_{j} \cdot r_{i j}\right) \quad j=\alpha_{1}, \quad \alpha_{2}, \quad \alpha_{3}, \quad \alpha_{4}, \quad \beta, \quad \gamma, i=1,2, \ldots n
\end{aligned}
$$

Finally, the network with the highest utility value is chosen as the switching access network as follows: 


$$
\mathbf{u}_{\text {Best }}=\operatorname{argmax}\left(\mathbf{u}_{\mathbf{j}}\right), \quad \mathbf{i}=1,2, \ldots \mathrm{n}
$$

\section{Simulation and analysis}

In order to verify the performance of the proposed access selection method (En-AHP), we simulated En-AHP on the Exata platform, comparing with, the satellite network priority algorithm (Simple) and the unmodified AHP algorithm. The simulation scenario follows a real ship distribution with a total of 29 ships, and other infrastructure network equipment. The moving speed of ship follows the random moving model, and the velocity distribution is from 6 to $13 \mathrm{~m} / \mathrm{s}$ with the moving granularity of $10 \mathrm{~m}$. The simulation time of the scene is $30 \mathrm{~s}$, and the application traffic type is constant bit rate (CBR). The data packet delivered by the application is 512 bytes, and the maximum number of packets is 100 .

As shown in Fig. 4, where the simulation time and the size of data packet remain unchanged, as the contract rate decreases, the total number of contracts is reduced, and the throughput of the two links shows a downward trend. When the transmission interval is $0.8 \mathrm{~s}$, the trend becomes flat, and the packet delivery rate decreases gradually with respect to the throughput per unit time. The throughput of En-AHP per unit time is more than Simple and AHP. The reason is the EN-AHP selects the ship ad hoc network, and the speed of base network in EN-AHP is faster than the Simple and the unimproved AHP, which select the satellite communication. As a result, the data processing capabilities of EN-AHP are superior to those of Simple and the unmodified AHP.

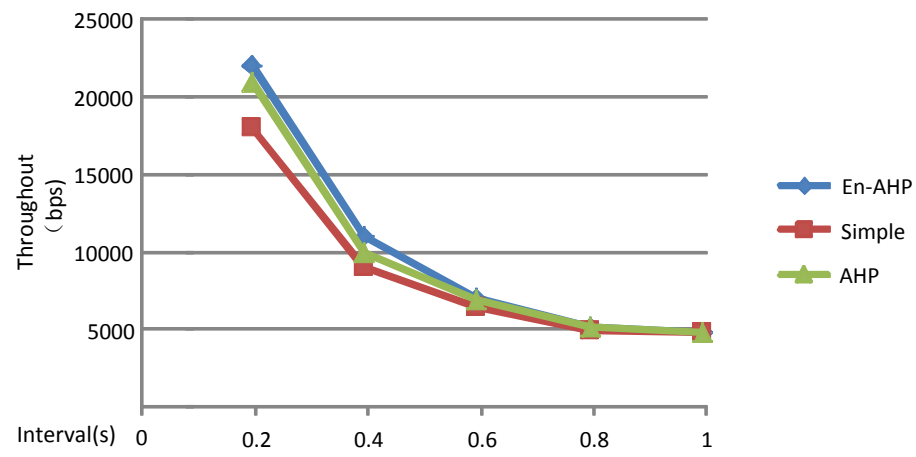

Fig.4. Comparison of throughput. The throughput shows a downward trend mainly.

As shown in Fig.5, the end-to-end delay of the Simple algorithm is always higher than that of EN-AHP. The primary variable is the transmission distance. In En-AHP, the data exchange between ships increases the delay, but the delay of Simple (satellite communication) is too large, accounting for the main proportion. With the reduce of packet sending rate, the end-to-end delay of Simple and unimproved AHP remain stable at $0.29 \mathrm{~s}$ basically, because for satellite, transmission distance of Simple algorithm is 
mainly composed of a satellite transmission path, and the characteristics of satellite communication is the delay remain stable.

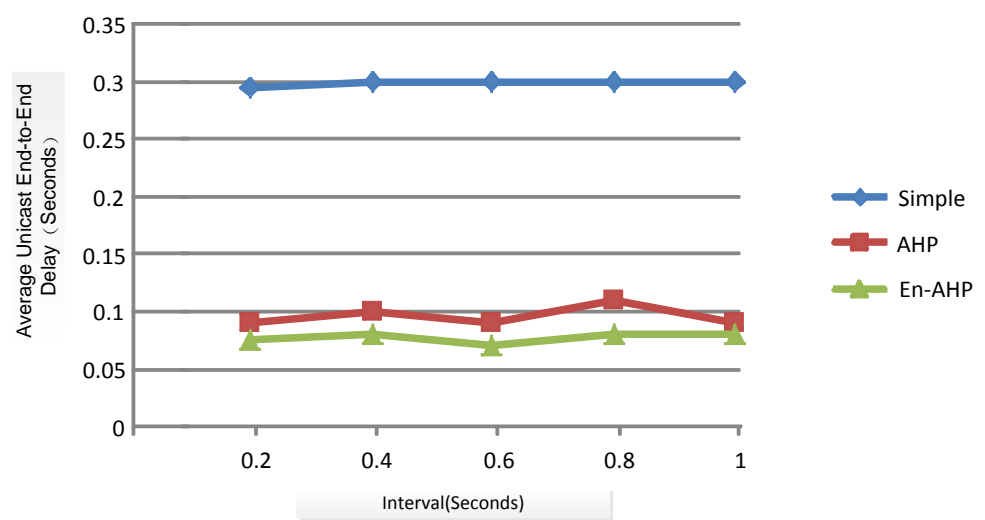

Fig.5. End-to-end delay. There is little difference between the En-AHP and AHP algorithm.

As shown in Fig.6, the Jitter of EN-AHP is always higher than Simple and unmodified AHP. Due to the number of ships is limited, the impact of link congestion is not considered. In EN-AHP, the movements of nodes in the ship self-organizing network lead to the instability of the link state, which is not as good as the satellite system. With the decrease of the packet transmission rate and the increase of the ship's moving distance, the data transmission jitter will intensify. The jitter of Simple remained stable, because the jitter of satellite communication is almost zero.

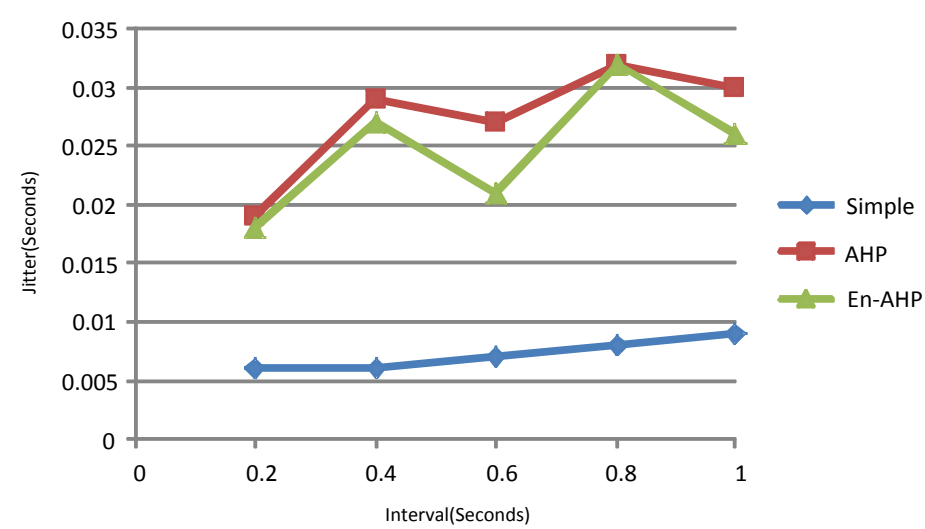

Fig.6. Comparison of Jitter. We found that the packet loss rate of EN-AHP is always higher than that of Simple and not improved AHP. The movement of the nodes in the ship's self-organizing network causes the link state to be unstable.

In terms of s the delay, jitter, and throughput, the benefits of EN-AHP are higher than Simple and unmodified AHP significantly. 


\section{Conclusion}

Multiple access networks may coexist in the Marine Internet. Considering the different preferences of users and different needs of different applications, we proposes an access network selection algorithm based on the composite weights. The algorithm is simulated on Exata, and the simulation results show that the network performance of the access network using the proposed algorithm is better with lower cost.

\section{References}

1. Jiang S.M. On the marine internet and its potential applications for underwater internetworking[C]. 8th ACM International Conference on Underwater Networks and Systems, 2013.

2. Sun Y.B. Research on heterogeneous network selection algorithms based on network performance and service feature perception [D]. Nanjing University of Posts and Telecommunications, 2012.

3. Yang G., Ji Y., Yu K., et al. General link layer technology in heterogeneous wireless access networks, [J]. radio engineering, 2005, 35 (10): 19-22.

4. Jia H.L. Research on access selection and admission control in heterogeneous wireless networks[D]. Zhejiang University, 2007.

5. Lv Q.F. Research on network selection algorithm based on entropy method and network analytic hierarchy process [D]. Inner Mongolia University, 2013.

6. Varma V. K, Ramesh S, Wong K D, et al. Mobility management in integrated UMTS/WLAN networks[C]// IEEE International Conference on Communications. 2003:1048 - 1053 .

7. Buddhikot M, Chandranmenon G, Han S, et al. Integration of 802.11 and thirdgeneration wireless data networks[J]. Physica C Superconductivity, 2003, 1(s 34):503--512.

8. Xu C.K, high M. T. Use LSA to reduce the dimensionality of the improved ART2 neural network clustering [J]. computer engineering and applications, 2014, (24): 133-138+177.

9. Zhou Q. P, Feng J. L. The model of port road monitoring based on shape mapping and attribute computing network [J]. modern computer, 2013, (09): 3-8. 